\title{
Path-pairability of infinite planar grids
}

\author{
Adam S. Jobson ${ }^{1}$ - André E. Kézdy ${ }^{1}$ - Jenő Lehel ${ }^{1,2}$
}

Accepted: 28 April 2020 / Published online: 21 May 2021

(c) The Author(s) 2021

\begin{abstract}
For a given integer $k \geq 1$, a graph $G$ with at least $2 k$ vertices is called $k$-path-pairable, if for any set of $k$ disjoint pairs of vertices, $s_{i}, t_{i}, 1 \leq i \leq k$, there exist pairwise edge-disjoint $s_{i}, t_{i}$-paths in $G$. The path-pairability numberis the largest $k$ such that $G$ is $k$-path-pairable. Bounds on the path-pairability number are given here if $G$ is the graph of infinite integer grids in the Euclidean plane. We prove that the path-pairability number of the integer quadrant is 4 , and we show that the integer half-plane is 6-path-pairable and at most 7-path-pairable.
\end{abstract}

Keywords Linkage $\cdot$ Infinite grid $\cdot$ Path-pairability $\cdot$ Interconnection networks

Mathematics Subject Classification $05 \mathrm{C} 10 \cdot 05 \mathrm{C} 38 \cdot 05 \mathrm{C} 40 \cdot 94 \mathrm{C} 15$

\section{Introduction}

Problems concerning edge-disjoint paths have several applications in the areas of routing, wavelength assignment, call admission control, point-to-point delivery, real-time communication, etc. (see [5]). In VLSI layout and reconfiguration problems the linkage properties are studied on a planar grid network, that is the underlying graph of the model is the Cartesian product of paths (see [1]). The general concept of path-pairability we are dealing with here is actually originated in a practical problem concerning telecommunications network as first described in [2]. Such a data- or telephone network is a collection of terminals (hosts), transmission links, and intermediate nodes which are assembled so as to enable simultaneous communication between pairs of terminals.

For a given integer $k \geq 1$, a graph $G$ with at least $2 k$ vertices is $k$-path-pairable, if for any set of $k$ disjoint pairs of vertices $s_{i}, t_{i}, 1 \leq i \leq k$, called terminals, there exist pairwise edge-

Jenő Lehel

lehelj@renyi.hu

Adam S. Jobson

adam.jobson@1ouisville.edu

André E. Kézdy

andre.kezdy@louisville.edu

1 Department of Mathematics, University of Louisville, Louisville, KY 40292, USA

2 Alfréd Rényi Institute of Mathematics, Budapest, Hungary 
disjoint $s_{i}, t_{i}$-paths in $G$. The path-pairability number, denoted $p p(G)$, is the largest $k$ such that $G$ is $k$-path-pairable. A summary of early results concerning path-pairability was given in [3], recent papers witness a renewed interest in linkage properties of Cartesian products of graphs (see [10-12]). The parameter $p p(G)$ was investigated recently in [6,7], for finite and infinite grid graphs $G$, that is, for the Cartesian product of finite or infinite paths.

We use here $\mathbb{N}$ and $\mathbb{Z}$ to denote the one-way and the two-way infinite path on the natural numbers and on the integers, respectively, with an edge between $i$ and $j$ if and only if $|i-j|=$ 1. The Cartesian product of two of these infinite paths lead to three kinds of infinite grid, $\mathbb{Z} \square \mathbb{Z}$ (integer plane), $\mathbb{N} \square \mathbb{Z}$ (integer half-plane), and $\mathbb{N} \square \mathbb{N}$ (integer quadrant). (Between vertices $(p, q)$ and $(i, j)$ of a product graph there is an edge if and only if $|p-i|+|q-j|=1$.)

In $[6,8]$ the path-pairability number of the finite rectangular grids, the Cartesian product $P_{a} \square P_{b}$, was determined for every pair of positive integers $a, b \geq 2$. From the meticulous proof of $p p\left(P_{6} \square P_{6}\right)=4$ in [8] we immediately obtain the path-pairability number of $\mathbb{N} \square \mathbb{N}$. In Sect. 4 a much shorter transparent proof is presented for $p p(\mathbb{N} \square \mathbb{N})=4$. We use two technical lemmas (Lemmas 4.1 and 4.2) whose detailed proofs are given in [9].

Determining the path-pairability number of the integer half-plane and the integer plane remain challenging open problems. The bounds $10 \leq p p(\mathbb{Z} \square \mathbb{Z}) \leq 14$ were obtained in [7]; here in Sect. 3 we prove $6 \leq p p(\mathbb{N} \square \mathbb{Z}) \leq 7$ by using similar methods. The lower bound proofs are based on Hall's matching theorem, the upper bound constructions violate cut conditions as explained in Sect. 2. Conjectures on the value of these path-pairability numbers, supported/tested by exhaustive computer search, are proposed in Sect. 5

\section{Proof strategies}

To prove that a graph $G$ is not $k$-path-pairable, we need to exhibit a counterexample consisting of $k$ terminal pairs that does not admit edge disjoint linkage. For the proof that no linkage exists we use a necessary condition, called cut condition ${ }^{1}$ and its variations. As an example, the bound $p p(\mathbb{Z} \square \mathbb{Z})<19$ follows from a pairing where one terminal from each pair is located at the vertices of a $4 \times 5$ grid $R$ and the second members of the pairs are located anywhere in $(\mathbb{Z} \square \mathbb{Z})-R$. Indeed, if there was a linkage for the 19 pairs, 19 edge disjoint paths must exit $R$, but there are only 18 edges between $R$ and $(\mathbb{Z} \square \mathbb{Z})-R$. We might say, there is no edge disjoint linkage because the cut condition is violated by this particular pairing. Sharper upper bounds can be obtained by using stronger necessary conditions derived from the cut condition. The best bound $p p(\mathbb{Z} \square \mathbb{Z})<15$ that we know so far was obtained in this way in [7].

Let $G$ be an infinite grid whose vertices are arranged into 'vertical' and 'horizontal' infinite lines (or half-lines). For a proof that $\operatorname{pp}(G) \geq k$, let $\pi_{i}=\left\{s_{i}, t_{i}\right\}$ be an arbitrary pairing of $2 k$ terminals in $G$. Denote by $T$ the set of those $2 k$ terminals and include $T$ into a (smallest) rectangle $R \subset G$. An edge disjoint linkage of the $k$ pairs can be obtained by a general two-stage procedure.

The first stage, the essential part of the proofs, is slightly different for each of the three infinite grids $\mathbb{Z} \square \mathbb{Z}, \mathbb{N} \square \mathbb{Z}$, and $\mathbb{N} \square \mathbb{N}$. First we take the edge disjoint linkage of some pairs inside $R$; then every unlinked terminal $u \in T$ is 'mapped' to a vertical or horizontal half-line $\ell(u)$ through $u$ going out from $R$ in such a way that all these half-lines are pairwise edge-

${ }^{1}$ If the vertex set of $G$ is partitioned into two sets, the number of edges of $G$ between the sets is not smaller than the cardinality of each of these sets. 
disjoint and disjoint from the paths used by the linkage inside $R$. To obtain such a mapping Hall's matching theorem (see [4]) will be used.

The second stage is common for each of the three infinite grids. We take $k$ pairwise disjoint finite paths $F_{i}$ in the grid, $1 \leq i \leq k$, each intersecting all the half-lines assigned to unlinked terminals by the mapping in the first stage. For every unlinked terminal $u \in \pi_{i}$, let $u^{*} \in \ell(u) \cap F_{i}$; then the linkage for any unlinked pair $\pi_{i}$ can be completed along $F_{i}$ between $s_{i}^{*}$ and $t_{i}^{*}$.

A humble application of Hall's matching theorem in the first stage would lead to small $k$, a plain lower bound on the path-pairability number of the grid. In order to achieve larger $k$, the application of Hall's matching theorem will be preceded with an investigation of exceptional terminal pairings, a few annoying cases called here 'obstructions', when Hall's condition is violated.

In order to handle the linkage of terminals of an obstruction, the general procedure is refined as follows. Besides linking a pair inside $R$ we are also allowed to 'move' a terminal into a 'mate' at a more favorable locations along pairwise edge-disjoint 'mating paths' in $R$. The first stage of the refined procedure starts with the edge disjoint linkage of some pairs inside $R$, then half-lines 'sticking out' from $R$ are assigned to the unlinked terminals. The head vertices of these half-lines located on the boundary of $R$ will serve as mates; to conclude the first stage edge-disjoint mating paths are built from each unlinked terminal to the head vertex of an appropriate half-line. In this way the unlinked terminals 'exit' through the halflines headed at their mates, and those pairs leaving $R$ become linked in the standard second stage as described above. The lower bound $p p(\mathbb{N} \square \mathbb{Z}) \geq 6$ in Proposition 3.2 is obtained in this way.

The process of selecting the pool of mates and the strategy of building non-conflicting mating paths from terminals to mates is tailored to the underlying grids under study and depends on specific conditions different from case to case (see [6-10]).

\section{The integer half-plane}

In this section we prove the bounds $6 \leq p p(\mathbb{N} \square \mathbb{Z}) \leq 7$

Proposition 3.1 The grid $\mathbb{N} \square \mathbb{Z}$ is not 8-path-pairable.

Proof Let $A(i), i=1,2, \ldots$, denote the horizontal lines (also called rows) of the grid $G=\mathbb{N} \square \mathbb{Z}$ from top down. Let $R \subset A(1) \cup A(2)$ be a $2 \times 5$ rectangle; locate 10 terminals $s_{1}, t_{1}, s_{2}, t_{2}, s_{i}, 3 \leq i \leq 8$, in $R$ as seen in Fig. 1 , and let $t_{j}, 3 \leq j \leq 8$, be placed arbitrarily in $G-R$.

Assume there is a linkage in $G$ for each pair $\pi_{i}, 1 \leq i \leq 8$. Observe that inside $R$ there is a linkage either for $\pi_{1}$ or for $\pi_{2}$, but not for both. Therefore, at least eight terminals must leave $R$ using the nine exits (two exits on $A(1)$ and $A(2)$, and one exit on each vertical half-line). By symmetry, we may assume that the $s_{1}, t_{1}$-path $P_{1}$ of the solution is contained by $R$. Observe that between $\left\{s_{1}, s_{2}\right\}$ and $R \backslash\left\{s_{1}, s_{2}\right\}$ there are two edges, and one is used by $P_{1}$. Thus at most two among the three exits at $s_{1}, s_{2}$ can be accessed by the remaining terminals. The same is true considering the three exits at $t_{1}, t_{2}$. Hence only seven terminals are able to leave $R$, a contradiction.

The vertices of a $p \times q$ rectangular grid $R$ are considered as entries of a $p \times q$ matrix, and $(i, j) 1 \leq i \leq p, 1 \leq j \leq q$, denotes the vertex in row $i$ and column $j$. The rows and the 
Fig. $1 p p(\mathbb{N} \square \mathbb{Z})<8$

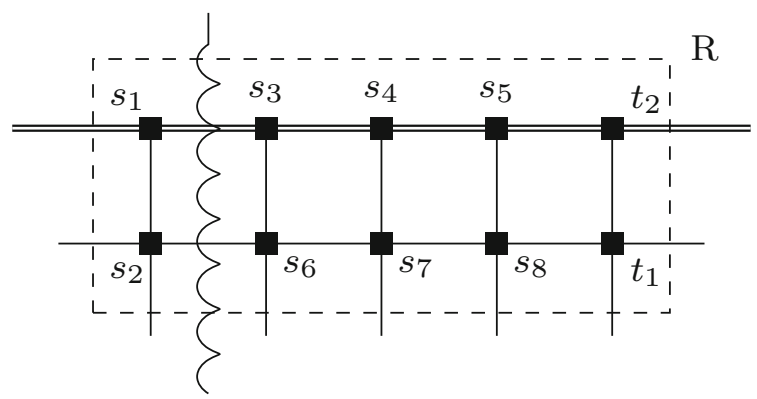

columns of $R$ are simply denoted by $A(i), 1 \leq i \leq p$, and $B(j), 1 \leq j \leq q$, respectively. The number of terminals in a finite subset $S \subset \mathbb{N} \square \mathbb{Z}$ is denoted by $\|S\|$.

\section{Proposition 3.2 The grid $\mathbb{N} \square \mathbb{Z}$ is 6-path-pairable.}

Proof Let $T$ be a set of at most 12 terminals in the grid $G=\mathbb{N} \square \mathbb{Z}$; let $L(T)$ be the set of all horizontal lines and vertical half-lines in $G$ containing a terminal. We wish to exhibit a mapping $\psi: T \longrightarrow L(T)$ such that $u \in \psi(u)$, for every $u \in T$, furthermore, at most one terminal is mapped to each vertical half-line of $L(T)$, and at most two terminals are mapped to each horizontal line of $L(T)$. Let $S \subseteq T$ and assume that $L(S)$ contains $p$ horizontal lines and $q$ vertical half-lines. The existence of the mapping $\psi$ would follow from Hall's condition provided

$$
|S| \leq \min \{12, p q\} \leq 2 p+q
$$

holds for every $S \subseteq T$.

A grid formed by any $p$ rows and any $q$ columns of $G$, and holding a set $S \subseteq T$ of terminals is considered to be an obstruction, provided $|S|>2 p+q$. We may successively reduce an obstruction to a minimal one as follows. If one or two terminals are contained in a row, then they are mapped into one half-line or two disjoint half-lines emanating from these terminals; if a single terminal is contained by a column, then it is mapped to the vertical half-line emanating from this terminal. After discarding the terminals and the assigned rows and columns the procedure is repeated until an obstruction is reduced to minimal.

Thus a (minimal) obstruction is defined as a $p \times q$ rectangle $R$ containing a set of terminals, $S \subseteq T$, such that each row has at least 3 terminals and each column has at least 2 terminals, furthermore, $|S|>2 p+q$. In particular, an obstruction satisfies $2 \leq p \leq 4$ and $3 \leq q \leq 6$.

Case a: $p=2$. Let $R_{q}$ be a minimal $2 \times q$ obstruction containing a set $S_{q}$ of terminals. Each column of $R_{q}$ has 2 terminals, thus we have $\left|S_{q}\right|=2 q \leq|T|=12$. Since $\left|S_{q}\right|=2 q \geq$ $1+(2 p+q)=q+5$, it follows that $q=5$ or 6 . Let $R \subset R_{q}$ be a $2 \times 4$ grid. Since $T$ contains 6 pairs, and $\|R\|=8$, there is a pair $\pi_{1} \subset R$, by the pigeon hole principle.

We label the vertices of $R$ along the 8-path $Q^{*}=\left(v_{1}, v_{2}, \ldots, v_{7}, v_{8}\right)$ as in the top left in Fig. 2. Let $P_{1} \subset Q^{*}$ be the (unique) linkage for $\pi_{1}$ along $Q^{*}$. For $j=1,8$ we define $Q_{j} \subset Q^{*}$ to be the mating path (possibly of length 0 ) from the terminal at $v_{j}$ to the closest end vertex of $P_{1}$. Then we assign to the unlinked terminals and mates the vertical half-lines at $v_{i}$, for $3 \leq i \leq 6$, and the two disjoint horizontal half-lines at $v_{2}$ and at $v_{7}$ (see the top right in Fig. 2).

For $q=5$, a mapping described above for a $2 \times 4$ subgrid $R \subset R_{5}$ can be extended with a vertical half-line disjoint from $R$ and a horizontal half-line in the second row of $R$ assigned to the two terminals not in $R$. For $q=6$, let $R \subset R_{6}$ be the $2 \times 4$ grid obtained by the 

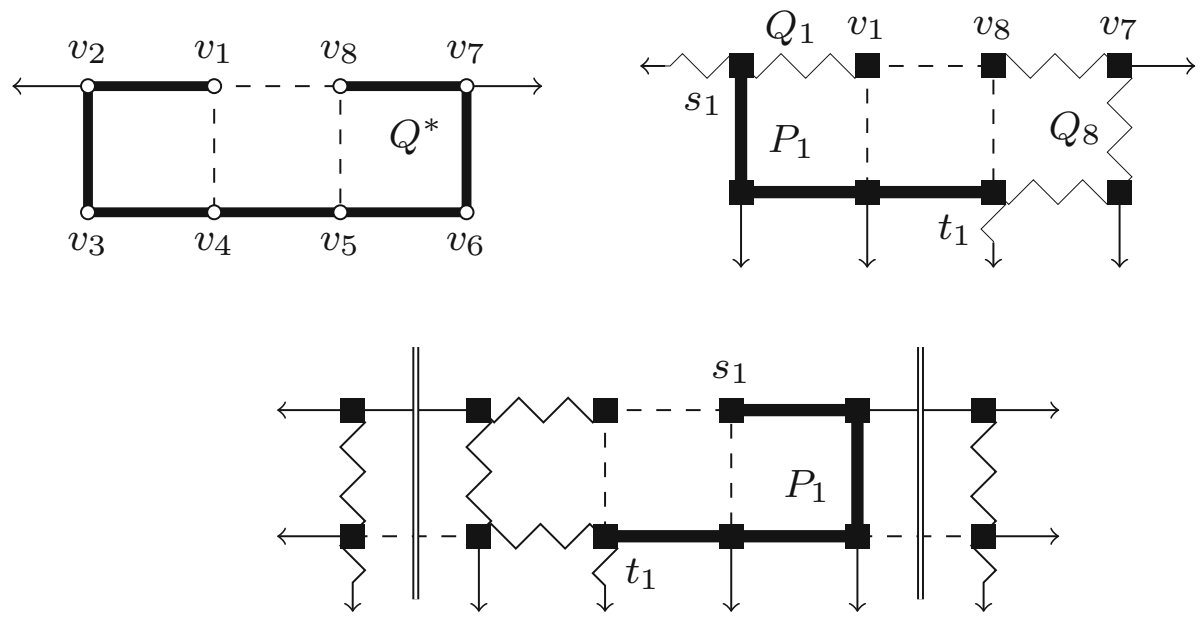

Fig. 2 Exit a $2 \times 4$ rectangle and exit a $2 \times 6$ obstruction
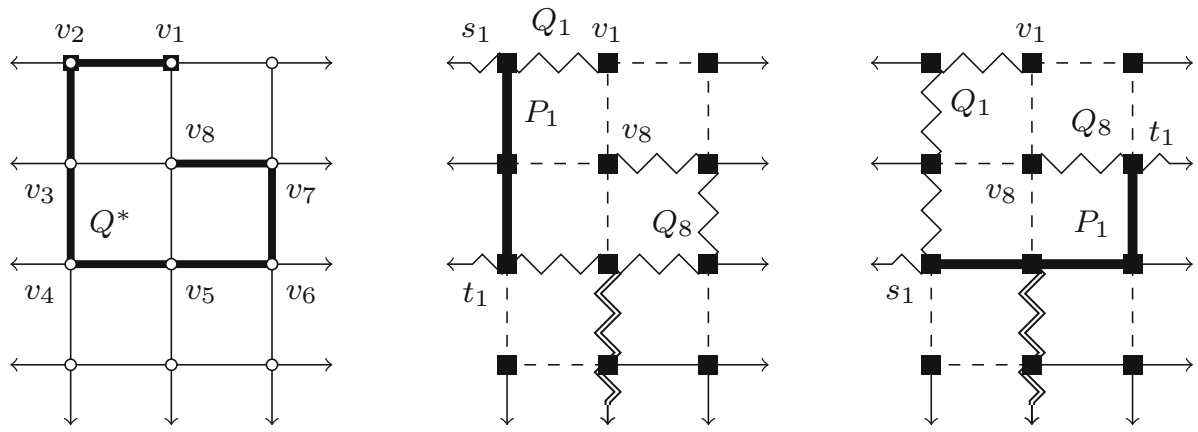

Fig. 3 Solving the $4 \times 3$ obstruction

removal of the first and last column from $R_{6}$. Then the mapping described for $R \subset R_{6}$ can be extended with two vertical half-lines disjoint from $R$ and two horizontal half-lines in the second row of $R_{6}$ assigned to the four terminals not in $R$ (see the third picture in Fig. 2).

Case b: $q=3$ and $p \geq 3$. Let $R_{p}$ be the $p \times 3$ minimal obstruction containing a set $S_{p}$ of terminals. Since $12 \geq\left|S_{p}\right|=3 p \geq 1+2 p+q=2 p+4$, we obtain $p=4$. Thus we assume that the only minimal obstruction is formed by 12 terminals located at the vertices of a $4 \times 3$ rectangle $R$. The strategy of handling the obstruction is similar to the one in Case a.

We label the vertices of $R \backslash A(4)$ along the 8-path $Q^{*}=\left(v_{1}, v_{2}, \ldots, v_{7}, v_{8}\right)$ as on the left in Fig. 3. There is a pair $\pi_{1} \subset Q^{*}$, by the pigeon hole principle. Let $P_{1} \subset Q^{*}$ be the (unique) linkage for $\pi_{1}$ along $Q^{*}$. For $j=1,8$, we define $Q_{j} \subset Q^{*}$ to be the mating path (possibly of length 0 ) from the terminal at $v_{j}$ to the closest end vertex of $P_{1}$. Then we assign to the unlinked terminals and mates in $Q^{*}$ the vertical half-line at $v_{5}$, and the disjoint horizontal half-lines at $v_{i}$ for $2 \leq i \leq 7, i \neq 5$. Terminals $(4,1),(4,3)$ are assigned to vertical half-lines, terminals $(1,3),(4,2)$ exit along horizontal half-lines going right, each emanating from them (see examples in Fig. 3).

Case c: $p=3, q=4$, and $|S|=11$ or 12 . Let $R$ be the $3 \times 4$ rectangle with a terminal at each vertex except possible one position. The strategy is similar to the ones in Case $a$ and $b$. 

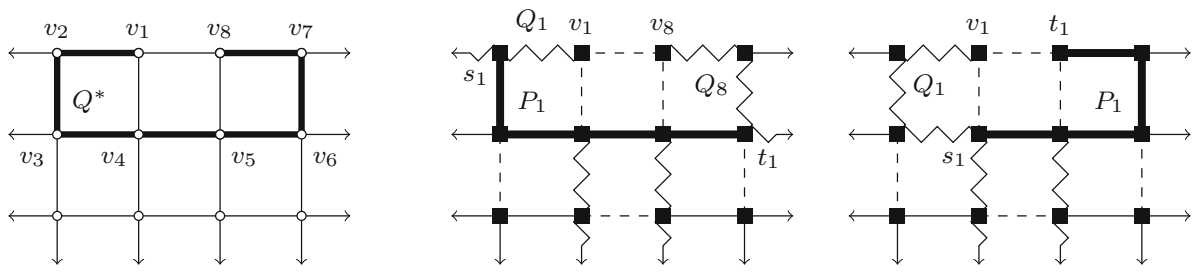

Fig. 4 Solving $3 \times 4$ obstructions

Fig. 5 Notations in Case d

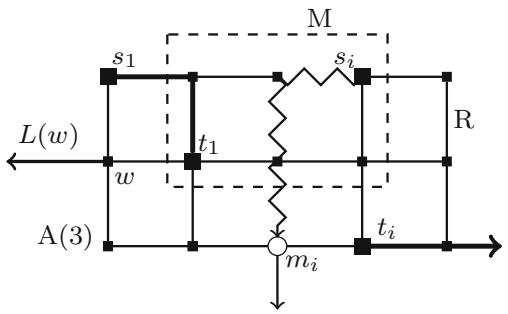

We label the vertices of $R \backslash A(3)$ along the 8-path $Q^{*}=\left(v_{1}, v_{2}, \ldots, v_{7}, v_{8}\right)$, see the left of Fig. 4. There is a pair $\pi_{1} \subset Q^{*}$ by the pigeon hole principle. Let $P_{1} \subset Q^{*}$ be the (unique) linkage for $\pi_{1}$ along $Q^{*}$. For $j=1,8$, we define $Q_{j} \subset Q^{*}$ to be the mating path from the terminal at $v_{j}$ to the closest end vertex of $P_{1}$. Then we assign to the unlinked terminals and mates in $Q^{*}$ the vertical half-lines at $v_{4}, v_{5}$, and the disjoint horizontal half-lines emanating from $v_{i}$ for $i \in\{2,3,6,7\}$. Vertical half-lines are assigned to terminals $(3,1),(3,4)$, and terminals $(3,2),(3,3)$ exit along disjoint horizontal half-lines emanating from them (see examples in Fig. 4).

There remain several minimal obstructions with parameters $p=3, q=5$, and $|S|=12$. Each column of a $3 \times 5$ rectangle $R$ contains at most one, and each row of $R$ contains at most two terminal-free vertices we will call holes. These obstructions are handled next in Case $\mathrm{d}$ and Case e, according to the number of holes in row $A(3)$ of $R$.

Let $M=\{(i, j) \mid 1 \leq i \leq 2 \leq j \leq 4\}$ be the middle rectangle containing the six interior vertices of $R$. A vertex $w \in R \backslash M$ is called a boundary vertex; each boundary vertex is the head of a half-line $L(w)$ sticking out from $R$. A terminal located at a boundary vertex of $R$ is called a boundary terminal.

Case d: $\|A(3)\|=3$. Since $A$ (3) contains two holes, there is a third hole in $R \backslash A(3)$. Depending on the location of the third hole, there are 6 or 7 boundary terminals to be associated with some of the 11 horizontal or vertical half-lines emanating from the boundary vertices. Then there remain 5 or 4 unassigned half-lines available to escape terminals from $M$. Our strategy consists of finding a linkage for an appropriate pair $\pi_{1} \subset R \backslash A$ (3) inside $R \backslash A(3)$ and 'move down' (at most) 4 further terminals to their mates $m_{i} \in A(3), 1 \leq i \leq 4$, (see Fig. 5).

First we discuss how to distribute the 7 horizontal and vertical half-lines at the vertices of $A(3)$ among the three terminals $t_{1}, t_{2}, t_{3} \in A(3)$ and four fixed mate positions $m_{1}, m_{2}, m_{3}, m_{4} \in A(3)$ not necesserly distinct from terminals (indexing is from left to right along $A(3)$ ). Terminals $t_{1}$ and $t_{3}$ are mated along $A(3)$ to be associated with disjoint horizontal half-lines emanating from $(3,1)$ and $(3,5)$, respectively. Furthermore, the vertical half-line at $m_{i}$ is associated to the terminal mated with $m_{i}$, for every $1 \leq i \leq 4$. We are able to finish the assignment of the half-lines in two particular cases as follows. 


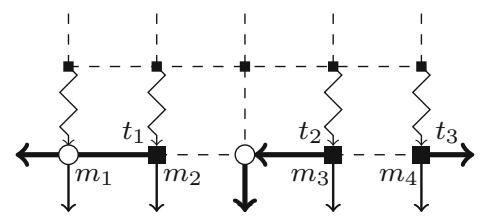

(i)

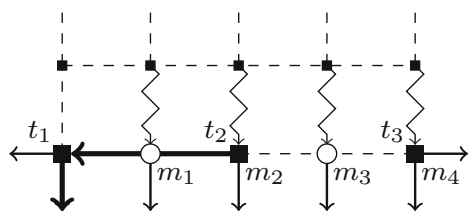

(ii)

Fig. 6 Assigning half-lines to terminals and mates in $A(3)$
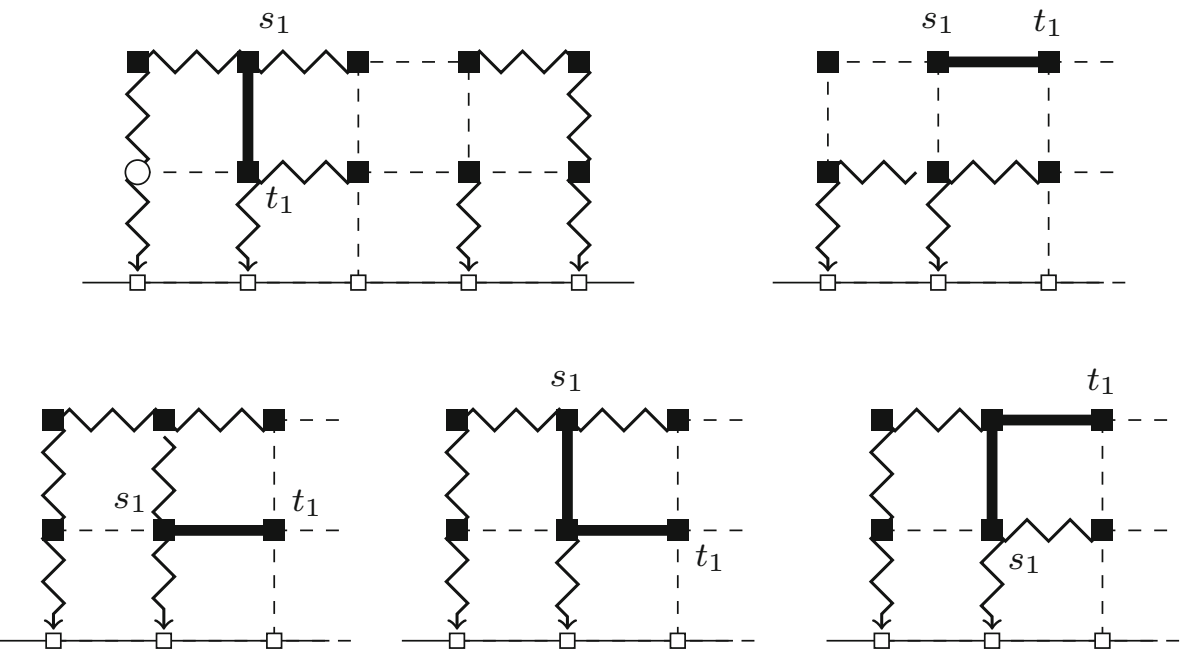

Fig. $7 \pi_{1} \subset B(2) \cup B(3)$

(i) If $(3,3) \neq m_{i}(i=2,3)$, then $t_{2}$ is mated to $(3,3)$ and is associated with the vertical half-line at $(3,3)$ (see Fig. 6 (i)).

(ii) If $t_{1}=(3,1)$ and $(3,1) \neq m_{1}$, then $t_{2}$ is mated to $(3,1)$ and the vertical half-line at $(3,1)$ is assigned to $t_{2}$ (see Fig. 6 (ii)).

(d.1) Assume there is a pair in $M$, say $\pi_{1} \subset M$. By symmetry, we have either $\pi_{1} \subset B(2)$, or $\pi_{1} \subset B(2) \cup B(3)$, or $\pi_{1} \subset B(2) \cup B(4)$. If $\pi_{1} \subset B(2)$ or $\pi_{1} \subset B(2) \cup B(3)$, there are five patterns for the 4 unlinked terminals. Mating for the terminals in $B(4)$ is common for them: $(1,4) \rightsquigarrow(3,5)$ and $(2,4) \rightsquigarrow(3,4)$, where the symbol $t \rightsquigarrow w$ means the mating of the terminal at location $t \in M$ into a mate at location $w \in A(3)$.

The mating paths for each is shown in Fig. 7 (the top left picture shows the solution for $\pi_{1} \subset B(2)$, the other ones are shown without repeating the identical columns $\left.B(4), B(5)\right)$.

For $\pi_{1} \subset B(2) \cup B(4)$ the terminals are mated according to $(1,3) \rightsquigarrow(3,1),(2,3) \rightsquigarrow$ $(3,5)$, furthermore, $(i, j) \rightsquigarrow(3, j)$, for $i=1,2, j=2,4$, and $(i, j) \notin \pi_{1}$. By symmetry, $\pi_{1}$ has three different locations, the mating paths are shown in Fig. 8. In each case $(3,3)$ is not assigned as a mate, thus the assignment satisfies (i).

(d.2) There is no terminal pair in $M$, and $M$ contains no hole. By symmetry, we may assume that the only hole in $R \backslash A(3)$ is $h \in\{(1,1),(2,1)\}$. By the pigeon hole principle, there is a terminal pair $\left(s_{1}, t_{1}\right)$ among the seven terminals of $(R \backslash(A(3) \cup B(3))$, furthermore, $s_{1} \in\{(2,1),(1,1)\}$ and $t_{1} \in M$. Notice that $(3,1)$ is a terminal.

The mating plan for the case when $s_{1}, h \in B(1) \backslash A(3)$ is as follows 

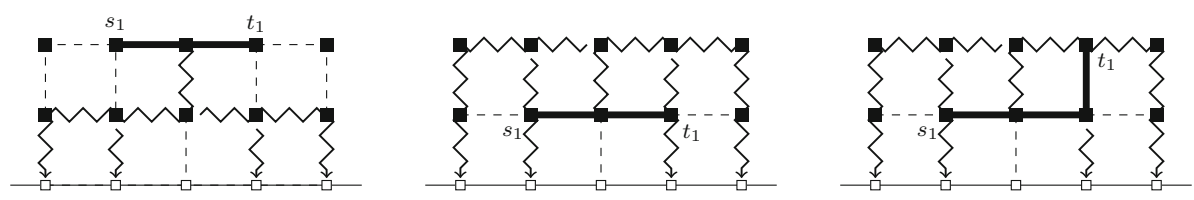

Fig. $8 \pi_{1} \subset B(2) \cup B(4)$
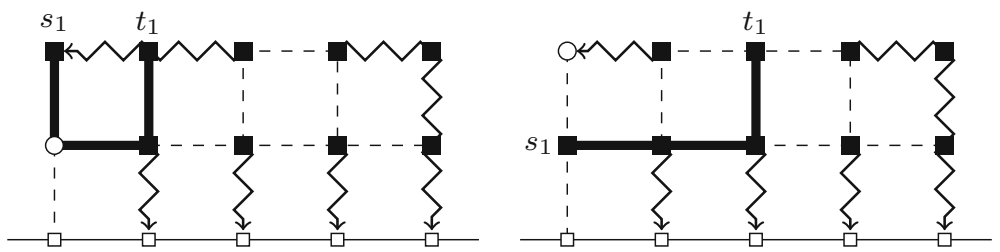

Fig. $9 s_{1}, h \in B(1) \backslash A(3)$
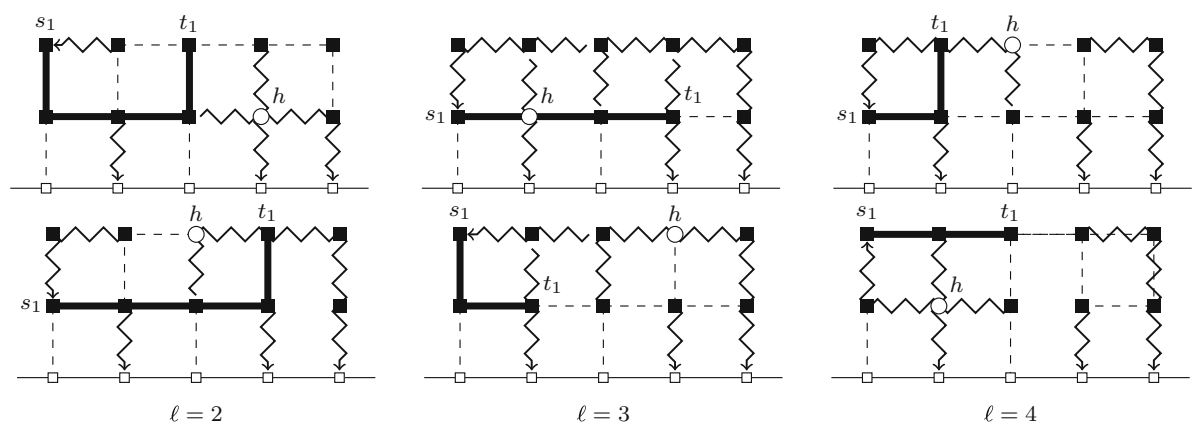

Fig. $10 s_{1} \in B(1)$ and $t_{1}, h \in M$

$$
\begin{aligned}
& (1,2) \rightsquigarrow(1,1),(1,3) \rightsquigarrow(3,5),(1,3) \rightsquigarrow(1,1),(1,4) \rightsquigarrow(3,5), \\
& (i, j) \rightsquigarrow(3, j) \text { for } i=1,2, j=2,3,4 \text { and }(i, j) \neq t_{1} .
\end{aligned}
$$

Notice that the left horizontal half-line emanating from $(1,1)$ is unused, because $(1,1) \in$ $\left\{s_{1}, h\right\}$, hence this location is an available mate in addition to those in $A(3)$. In each case the mating satisfies the half-line assignment (ii). The mating paths for the 12 possible patterns of $\pi_{1}$ are very similar, Fig. 9 shows two examples.

(d.3) Assume there is no terminal pair in $M$, and $h \in M$ is a hole. The strategy is similar to (d.2), it starts with linking a pair $\pi_{1}=\left(s_{1}, t_{1}\right)$ such that $t_{1} \in M$ and $s_{1} \in(B(1) \cup B(5)) \backslash A(3)$. We mate the other 4 terminals from $M$ to $A(3) \backslash\{(3,3)\}$, in order to satisfy the assignment in (i).

If $h \in B(j), 2 \leq j \leq 4$, then w.l.o.g. we may assume that there exists a pair $\pi_{1}=\left(s_{1}, t_{1}\right)$ such that $s_{1} \in\{(1,1),(2,1)\}$ and $t_{1} \in(R \backslash B(j)) \cap M$, since $\|(R \backslash B(j)) \cap M\|=4$ and $\|A(3)\|=3$. Let $t_{1} \in B(k), j \neq k$, and set $\ell \in\{2,3,4\} \backslash\{j, k\}$. Notice that $M$ has two terminals in $B(\ell)$ to mate, and one terminal from each of $B(j)$ and $B(k)$. According to the values $\ell=2,3$, or 4 , we propose the following mating plans, which have realizations with mating paths in each case.

For $\ell=2:(1,2) \rightsquigarrow s_{1},(2,2) \rightsquigarrow(3,2)$,

$(i, 3) \rightsquigarrow(3,5)$, where $i=1,2$ and $(i, 3) \notin\left\{h, t_{1}\right\}$,

$(i, 4) \rightsquigarrow(3,4)$, where $i=1,2$ and $(i, 4) \notin\left\{h, t_{1}\right\}$. 
For $\ell=3:(1,3) \rightsquigarrow s_{1},(2,3) \rightsquigarrow(3,5)$,

$(i, 2) \rightsquigarrow(3,2)$, where $i=1,2$ and $(i, 2) \notin\left\{h, t_{1}\right\}$,

$(i, 4) \rightsquigarrow(3,4)$, where $i=1,2$ and $(i, 4) \notin\left\{h, t_{1}\right\}$.

For $\ell=4:(1,4) \rightsquigarrow(3,5),(2,4) \rightsquigarrow(3,4)$,

$(i, 2) \rightsquigarrow(3,2)$, where $i=1,2$ and $(i, 2) \notin\left\{h, t_{1}\right\}$,

$(i, 3) \rightsquigarrow s_{1}$, where $i=1,2$ and $(i, 3) \notin\left\{h, t_{1}\right\}$.

Figure 10 shows examples of realizing the mating plan, two for each value of $\ell$. This concludes the proof of Case $\mathrm{d}$ when $\|A(3)\|=3$. For the proof of the last case $\|A(3)\| \geq 4$ we need a technical lemma.

Lemma 3.3 Let $D \subset \mathbb{N} \square \mathbb{Z}$ be a $2 \times 4$ rectangle of eight terminals labeled as below.

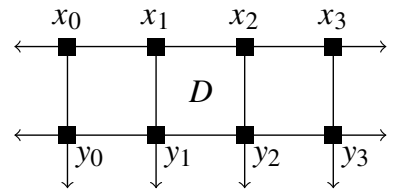

Assume that there is a pair $\pi_{1}$ among the terminals of $D$. If $y_{0} \notin \pi_{1}$, then there is a linkage in $D$ for $\pi_{1}$, and there exist edge disjoint mating paths mating the other six terminals to the head of distinct horizontal and vertical half-lines exiting D subject to either one of the following restrictions:

(A) The horizontal left half-line at $x_{0}$ is not assigned to any terminal.

(B) The horizontal half-lines at $y_{0}$ and $y_{3}$ are not assigned to any terminal, furthermore, edges $x_{1} y_{1}$ and $x_{2} y_{2}$ are not used by the linkage and by any mating paths.

Proof (A) If $x_{0} \notin \pi_{1}$, then we assign to the terminal $x_{0}$ the vertical half-line at $x_{0}$ and we assign to $y_{0}$ the left horizontal half-line at $y_{0}$. To complete a solution as required by (A) we consider the 6-path $Q^{*}=\left(x_{1}, y_{1}, y_{2}, y_{3}, x_{3}, x_{2}\right)$ and let $P_{1} \subset Q^{*}$ be the (unique) linkage in $Q^{*}$ for $\pi_{1}$.

For $x_{j} \notin \pi_{1}, j=1,2$, we define $Q_{j} \subset Q^{*}$ to be the mating path from the terminal at $x_{j}$ to the closest end vertex of $P_{1}$. Then we assign to the four terminals and mates in $Q^{*}-\pi_{1}$ the three vertical half-lines at $y_{i}$, for $1 \leq i \leq 3$, and the horizontal right half-line at $x_{3}$ (see examples in Fig. 11).

Among the six possible cases when $s_{1}=x_{0}$ and $t_{1}=x_{i}$ or $y_{i}, 1 \leq i \leq 3$, we show a solution for $t_{1} \in\left\{y_{1}, x_{2}, y_{2}\right\}$ in Fig. 12. Obvious variations of these examples by extending or shortening $P_{1}$ lead to a solution for $t_{1} \in\left\{x_{1}, x_{3}, y_{3}\right\}$.

(B) Let $Q^{*}=\left(x_{1}, x_{0}, y_{0}, y_{1}, y_{2}, y_{3}, x_{3}, x_{2}\right)$ and let $P_{1} \subset Q^{*}$ be the (unique) linkage for $\pi_{1}$ along $Q^{*}$. For $x_{j} \notin \pi_{1}, j=1,2$, we define $Q_{j} \subset Q^{*}$ to be the mating path from the terminal at $x_{j}$ to the closest end vertex of $P_{1}$. Then we assign to the six terminals and mates the four vertical half-lines at $y_{i}, 0 \leq i \leq 3$, and the two disjoint horizontal half-lines at $x_{0}$ and at $x_{3}$ (see examples in Fig. 13).

Case e: $\|A(3)\| \geq 4$. Assume that each row of the $3 \times 5$ rectangle $R$ contains one hole, and let $x=(2, a)$ and $y=(3, b)$ be holes for some $a \neq b$ and $1 \leq a, b \leq 5$. Then mate $(1, a)$ to $x$ and $(1, b)$ to $y$ along their columns. There remain two terminals in the top line $A(1)$, we assign to these terminals disjoint horizontal half-lines emanating from them. By symmetry, we may assume that $b \in\{1,3,4\}$. Let $D=R \backslash(A(1) \cup B(1))$ be the $2 \times 4$ grid with vertices labeled as in Lemma 3.3. Among the six pairs of terminals (and mates) eight 
Fig. 11 Solutions for (A) for $x_{0}, y_{0} \notin \pi_{1}$
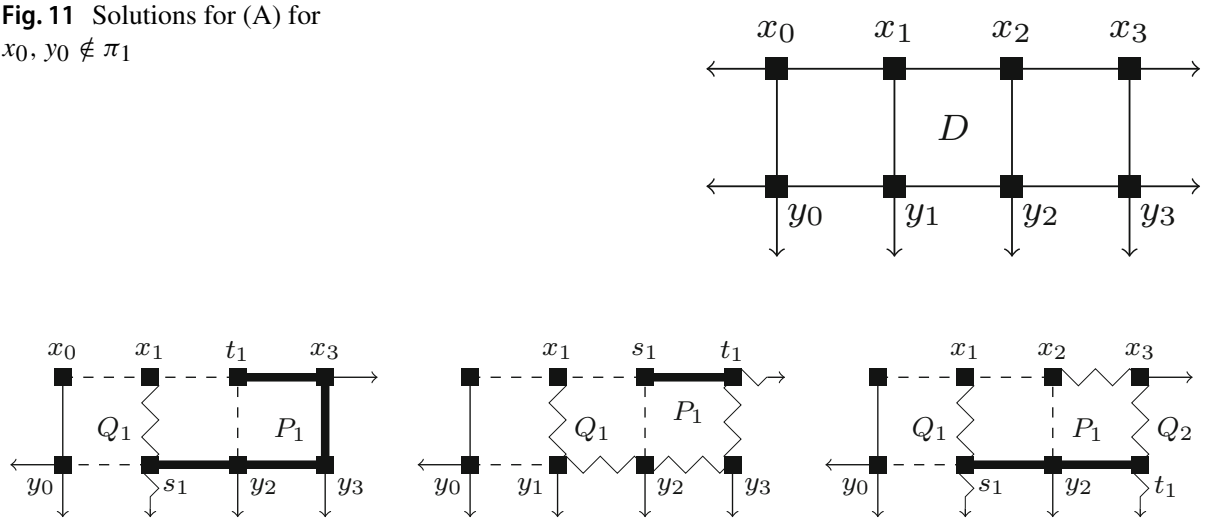

Fig. 12 Solutions for (A) with $x_{0} \in \pi_{1}, y_{0} \notin \pi_{1}$
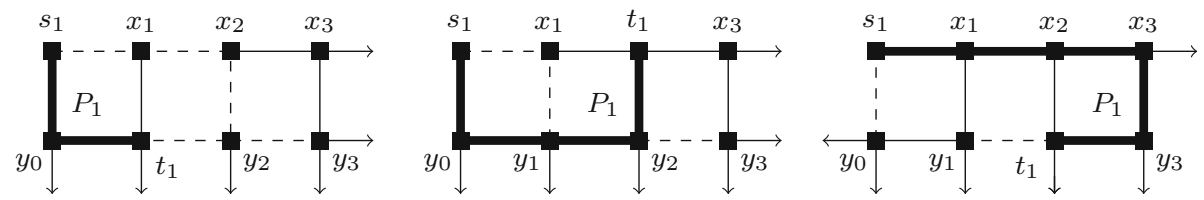

Fig. 13 Solutions for (B)

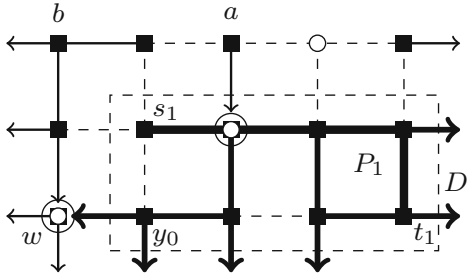

(A)

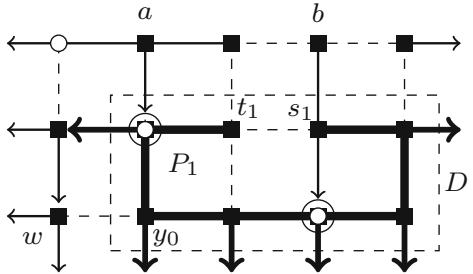

(B)

Fig. 14 Each row contains a hole (Case e)

are located at the vertices of $D$, thus there are at least two pairs in $D$; one of them, say $\pi_{1}$, does not contain $y_{0}$.

For $b=1$ we obtain a half-line assignment by Lemma 3.3 (A) which can be completed by a horizontal left half-line at $(2,1)$ and a vertical half-line at $(3,1)$. For $b=3$ or 4 we use Lemma 3.3(B); in each case the assignment (B) can be completed by the horizontal left half-line at $(3,1)$ and the vertical half-line at $(2,1)$. Figure 14 shows examples for $a=3$, $b=1$ and for $a=2, b=4$.

A solution is obtained similarly if row $A(\ell)$ contains two holes, where $\ell=1$ or 2 . Let the third hole in $R$ be $w=(i, b)$, where $i \neq \ell$ and $b \in\{1,3,4\}$ (w.l.o.g., by symmetry). We mate $(\ell, b)$ to $w$, and we assign disjoint horizontal half-lines emanating from the remaining two terminals in $A(\ell)$. Then we apply Lemma 3.3(A) or (B) with $D=R \backslash(A(\ell) \cup B(1)$ ) as before. Examples for (A) and (B) are presented in Fig. 15, where in picture (B) $\ell=2, b=4$ and $t_{1}=(2,4)$. This concludes the proof of Case $\mathrm{e}$, and the proposition follows. 


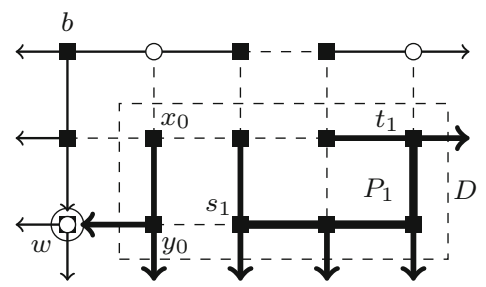

(A)

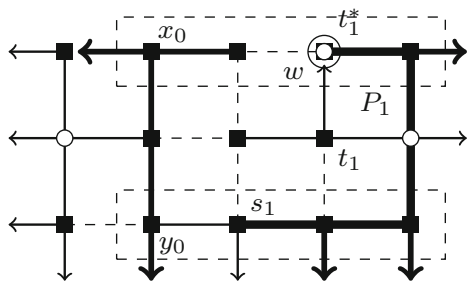

(B)

Fig. 15 Reducing a $3 \times 5$ obstruction to a $2 \times 4$ grid

Fig. $16 p p(\mathbb{N} \square \mathbb{N})<5$

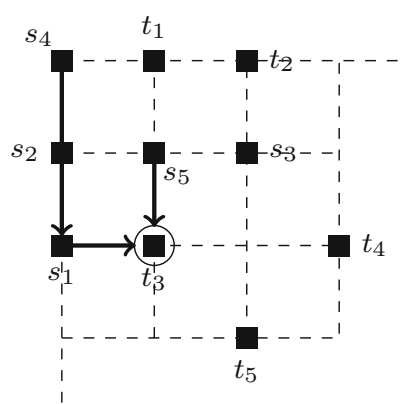

\section{The integer quadrant}

In this section we sketch a proof for $p p(\mathbb{N} \square \mathbb{N})=4$. We need two technical lemmas applied in [8] for determining the path-pairability number of the $6 \times 6$ grid $P_{6} \square P_{6}$. These lemmas, called there 'exit lemmas', serve to handle the corner of $G=\mathbb{N} \square \mathbb{N}$ crowded with terminals; their detailed proofs are presented in [9].

Let $Q$ be a $3 \times 3$ subgrid of $G$ with a horizontal bottom line $A$ and a vertical right boundary line $B$. The 5 vertices of $A \cup B$ are called exit vertices of $Q$. Recall that mating a terminal $t$ to a vertex $t^{\prime} \in G$ means fixing a $t, t^{\prime}$-path called a mating path. In the lemmas below $T=\cup\left\{\pi_{i} \mid 1 \leq i \leq 4\right\}$ is a set of 8 terminals (members of 4 pairs) located in $G$; and $\|Q\|=|T \cap Q|$ denotes the number of terminals in $Q$.

Lemma 4.1 [9] If $\|Q\|=7$ or 8 , then there is a linkage of two or more pairs in $Q$, and there are edge disjoint mating paths for the unlinked terminals to distinct exit vertices of $A \cup B$.

Lemma 4.2 [9] If $\|Q\|=6$, then there is a linkage of one or more pairs in $Q$, and there are edge disjoint mating paths for the unlinked terminals to distinct exit vertices of $A \cup B$ such that $B \backslash A$ contains at most one exit vertex.

Proposition $4.3 p p(\mathbb{N} \square \mathbb{N})=4$.

Proof The bound $p p(\mathbb{N} \square \mathbb{N})<5$ follows from the pairing of 10 terminals in Fig. 16. If there was a solution $P_{i}, 1 \leq i \leq 5$, then the path $P_{5}$ starting from terminal $s_{5}$ will either head right or down from $s_{5}$; without loss of generality assume it heads down to $t_{3}$. Either $P_{4}$ from $s_{4}$ or $P_{2}$ from $s_{2}$ will have to exit the upper-left $2 \times 2$ cluster along the edge down toward $s_{1}$. From that location either this path or the path $P_{1}$ starting from $s_{1}$ will then be forced to head 

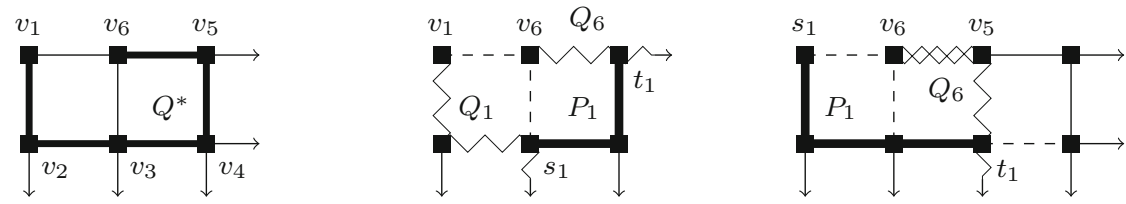

Fig. 17 Exit from a $2 \times 3$ or a $2 \times 4$ grid

right to $t_{3}$. There are now three different paths at that location but only two edges go out, a contradiction.

Next we verify that $G=\mathbb{N} \square \mathbb{N}$ is 4-path-pairable. Let $T$ be a set of 8 distinct terminals in $G$ partitioned into pairs $\pi_{i}, 1 \leq i \leq 4$. Let $L(T)$ be the set of all horizontal and vertical half-lines in $G$ containing a terminal. If a half-line $\ell \in L(T)$ contains just one terminal, then assign $\ell$ to this terminal, then remove it from $T$. Repeating this reduction reduces $T$ to a subset of terminals $S \subset T$ contained by a $p \times q$ rectangle $R$ such that each row and column of $R$ contains either zero or at least two terminals. We continue 'exiting' terminals from $R$ by mating interior terminals to free half-lines starting at the boundary of $R$, meanwhile, we are allowed (or urged) to link appropriate pair(s) in $R$. The linkage of the pairs is completed using the second stage of the procedure described in Sect. 2. Rows and columns with no terminal can be discarded, thus w.l.o.g. we my assume that each row and column of $R$ contains at least two terminals. Consequently we have $2 \leq p, q \leq 4$, and $|S| \leq \min \{8, p q\}$. By symmetry, we may assume that $p \leq q$.

If $q=2$, then $R$ is a $2 \times 2$ square and $|S|=4$. Then there are four edge disjoint half-lines in $G$ emanating from the four terminals. Thus $q>2$ can be assumed.

If $p=4$, then $q=4$, and thus $\|\ell\|=2$, for every $\ell \in L(S)$. Consider the 2-regular $4 \times 4$ bipartite graph $H$ where one partite set corresponds to the rows and the other partite set corresponds to the columns formed by the lines in $L(S)$, and the edges of $H$ correspond to the terminals in $S$. Then $H$ is the union of even cycles. For each even cycle we define disjoint half-lines emanating from consecutive terminals by assigning alternately horizontal and vertical half-lines in $G$. The remaining cases are: $p=2$ or $p=3$.

Case a: $p=2$. Since each column of $R$ has two terminals, we have $q=3$ or 4 .

The left picture of Fig. 17 shows the 6-path $Q^{*}=\left(v_{1}, \ldots, v_{6}\right)$ covering the terminals in the first three columns of $R$. The path $Q^{*}$ contains a pair $\pi_{1}$, by the pigeon hole principle. Let $P_{1} \subset Q^{*}$ be the (unique) linkage for $\pi_{1}$ in $Q^{*}$. For $v_{j} \notin \pi_{1}, j=1,6$, we define $Q_{j} \subset Q^{*}$ to be the mating path from the terminal at $v_{j}$ to the closest end vertex of $P_{1}$. Then we assign to the unlinked four terminals and mates the three vertical half-lines at $v_{i} \notin \pi_{1}$, for $2 \leq i \leq 4$, and the horizontal right half-line at $v_{5}$, provided $v_{5} \notin \pi_{1}$. For $q=4$ there are two more terminals in $B(4)$, they are assigned to the vertical half-line $B(4)$, and the horizontal right half-line in $A(2)$ (see examples in Fig.17).

Case b: $p=3$. Let $Q$ be the $3 \times 3$ square formed by the rows and the first three columns of $R$. Let $A=A(3) \cap Q$ and $B=B(3) \cap Q$. Since each column contains two or more terminals, we have $\|Q\| \geq 6$.

We apply Lemma 4.1 for $\|Q\|=7$ or 8 , and we apply Lemma 4.2 , for $\|Q\|=6$. For $q=3$ we assign half-lines to the at most four exits (mates and unlinked terminals) in $A \cup B$ among the the three vertical and three horizontal right half-lines emanating from $A \cup B$. For $q=4$, since each column of $R$ has two mates, we have $\|Q\|=6$. The vertical half-lines emanating from $A$ are assigned to the exits in $A$, and a horizontal half-line is assigned to the only exit in $B \backslash A$. The two terminals in $B(4)$ can be mated easily to the head of the two unused horizontal half-lines emanating from $B(4)$ and the vertical half-line in $B(4)$. 


\section{Conclusion}

It was proved in [7] that the grid $\mathbb{Z} \square \mathbb{Z}$ is 10 -path-pairable and not 15-path-pairable. These bounds and those proved here in Propositions 3.1, 3.2 and 4.3 are summarized as follows:

$$
p p(\mathbb{N} \square \mathbb{N})=4, \quad 6 \leq p p(\mathbb{N} \square \mathbb{Z}) \leq 7, \quad 10 \leq p p(\mathbb{Z} \square \mathbb{Z}) \leq 14 .
$$

The strategy of applying Hall's theorem (as in the proof of Proposition 3.2) is well-suited for deriving sharper lower bounds by using computer to resolve the rapidly increasing number of obstructions. A computer program confirmed that the half-plane is 7-path-pairable, thus we are making the 'safe conjecture' that $p p(\mathbb{N} \square \mathbb{Z})=7$. For the plane the upper and lower bounds are improved by a computer program to $11 \leq p p(\mathbb{Z} \square \mathbb{Z}) \leq 12$. We think that $p p(\mathbb{Z} \square \mathbb{Z})=12$ is the right value, meanwhile, computer checking whether the plane is 12-path-pairable is beyond our computing capacity.

Funding Open access funding provided by ELKH Alfréd Rényi Institute of Mathematics.

Open Access This article is licensed under a Creative Commons Attribution 4.0 International License, which permits use, sharing, adaptation, distribution and reproduction in any medium or format, as long as you give appropriate credit to the original author(s) and the source, provide a link to the Creative Commons licence, and indicate if changes were made. The images or other third party material in this article are included in the article's Creative Commons licence, unless indicated otherwise in a credit line to the material. If material is not included in the article's Creative Commons licence and your intended use is not permitted by statutory regulation or exceeds the permitted use, you will need to obtain permission directly from the copyright holder. To view a copy of this licence, visit http://creativecommons.org/licenses/by/4.0/.

\section{References}

1. W.-T. Chan, F.Y.L. Chin, H.-F. Ting, Escaping a grid by edge-disjoint paths. Algorithmica 36, 343-359 (2003)

2. L. Csaba, R.J. Faudree, A. Gyárfás, J. Lehel, R.H. Schelp, Networks communicating for each pairing of terminals. Networks 22, 615-626 (1992)

3. R.J. Faudree, Properties in pairable graphs. N. Z. J. Math. 21, 91-106 (1992)

4. P. Hall, On representation of subsets. J. Lond. Math. Soc. 10, 26-30 (1935)

5. C-C. Hsu, A genetic algorithm for maximum edge-disjoint paths problem and its extension to routing and wavelength assignment problem. Ph.D. thesis, North Carolina State University, 2013

6. A.S. Jobson, A.E. Kézdy, J. Lehel, On path-pairability of the finite grids. Eur. J. Math. 5, 1352-1363 (2019)

7. A.S. Jobson, A.E. Kézdy, J. Lehel, Linkage on the infinite grid. Inf. Process. Lett. 137, 51-56 (2018)

8. A.S. Jobson, A.E. Kézdy, J. Lehel, The $6 \times 6$ grid is 4-path-pairable. 2017. arXiv:1708.05407

9. A.S. Jobson, A.E. Kézdy, J. Lehel, Escaping from the corner of a grid by edge-disjoint paths. 2017. arXiv: 1708.05413

10. A.S. Jobson, A.E. Kézdy, J. Lehel, G. Mészáros, The path-pairability number of products of stars. Discuss. Math. Graph Theory 39, 909-924 (2019)

11. G. Mészáros, On path-pairability in the Cartesian product of graphs. Discuss. Math. Graph Theory 36, 743-758 (2016)

12. G. Mészáros, Linkedness and path-pairability in the Cartesian product of graphs. Ph.D. thesis at CEU, Budapest, 2015

Publisher's Note Springer Nature remains neutral with regard to jurisdictional claims in published maps and institutional affiliations. 